\title{
Morphological properties of massive galaxies at high $-z$ from GOODS
}

\author{
Swara Ravindranath ${ }^{1}$, E. Daddi ${ }^{2}$, M. Giavalisco ${ }^{3}$, H. C. Ferguson ${ }^{4}$ \\ and M. E. Dickinson ${ }^{5}$ \\ ${ }^{1}$ Inter-University of Astronomy \& Astrophysics, Pune, Maharashtra, India \\ email: swara@iucaa.ernet.in \\ ${ }^{2}$ CEA Saclay/Service d'Astrophysique, Gif-sur-Yvette Cedex, France \\ email: edaddi@cea.fr \\ ${ }^{3}$ University of Massachusetts, Amherst, Massacheussetts, USA \\ email: mauro@astro.umass.edu \\ ${ }^{4}$ Space Telescope Science Institute, Baltimore, Maryland, USA \\ email: ferguson@stsci.edu \\ ${ }^{5}$ National Optical Astronomy Observatory, Tucson, Arizona, USA \\ email: med@noao.edu
}

\begin{abstract}
We have used the $B z K$-selection to identify a composite population of passive, and star-forming galaxies at redshifts $1.4 \leqslant z \leqslant 2.5$ from the Great Observatories Origins Deep Survey (GOODS). Using an unprecedented large sample of galaxies in this redshift range, we characterize the morphological diversity through the analysis of the surface-brightness profile shapes for 171 galaxies with passive SEDs, and 1068 star-forming galaxies. We find that the $z \sim 2$ galaxies display a wide range of morphologies, from spheroidals to disk-like. Interestingly, the galaxies with passively-evolving SEDs predominantly have steep profiles as seen for the classical bulges at low redshifts, although they are very compact with $r_{e}<3 \mathrm{kpc}$. The starforming galaxies on the other hand exhibit mostly disk-like and merger morphologies, and have sizes comparable to their low $-z$ counterparts. Our results emphasize the need for an unbiased selection in order to reveal the morphological diversities, and range of galaxy properties at high redshifts.
\end{abstract}

Keywords. surveys, galaxies: bulges, galaxies: evolution, galaxies: formation, galaxies: highredshift

\section{Introduction}

Over the last decade, many deep surveys done with the Hubble Space Telescope have revealed that the range of galaxy morphologies seen in the local Universe was already in place by $z \sim 1$ (Lilly et al. 1998; Stanford et al. 2004; Ravindranath et al. 2004; Barden et al. 2005). At high redshifts, many studies have focused on the galaxy morphologies at $z>2.5$ because the Lyman-break technique offers an efficient and robust way to select large samples at these redshifts from the multi-wavelength, deep surveys (Giavalisco et al. 1996; Lotz et al. 2006 (L06); Ravindranath et al. 2006 (R06)). The morphological mix of the $z>2.5$ LBGs in the rest-frame UV typically consists of $\sim 30 \%$ bulge-like, and $\sim 40-50 \%$ of disk-like morphologies which also includes minor mergers, and about 20 $30 \%$ of the LBGs may be undergoing major mergers (R06; L06).

The redshifts, $1.4<z<2.5$, is particularly interesting because it marks the transition from the pre-dominantly irregular or distorted morphologies at $z>2.5$ to the regular bulge-disk morphology at $z<1$. This redshift range has been relatively less explored 

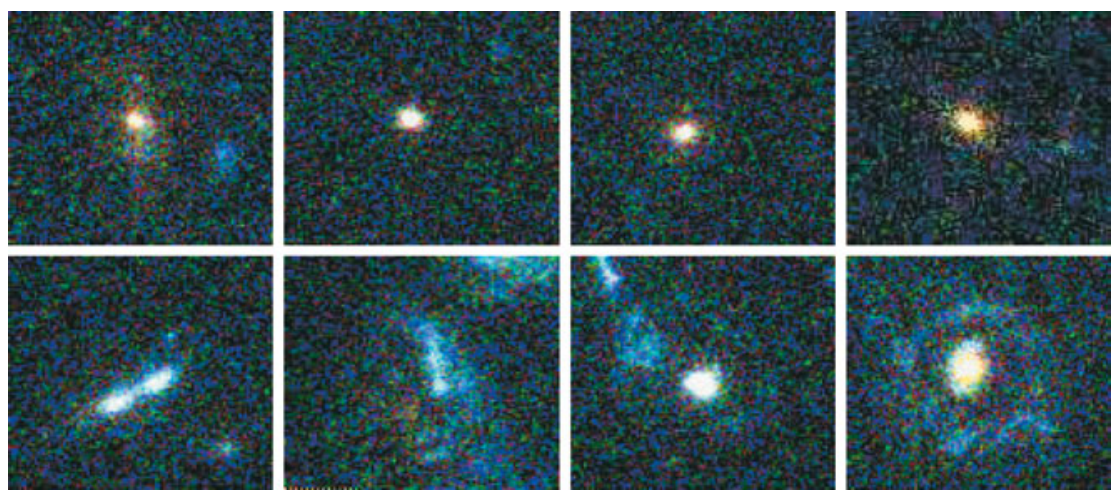

Figure 1. The $V, i, z$ color composite images showing the wide ranges of morphologies seen among the $B z K$ galaxies at $1.4 \lesssim z \lesssim 2.5$. The top panel shows examples of galaxies with passively-evolving SEDs, and the bottom panel shows galaxies with star-forming SEDs.

due to the difficulty in obtaining robust samples. However, with the recent deep, nearinfrared imaging and spectroscopic surveys, various schemes have been proposed for color selection of galaxies with $1.4<z<2.5$, for example, the distant red galaxies (DRGs) selection (Franx et al. 2003), and the $B z K$ selection (Daddi et al. 2004).

We have used the optical $(B, V, i$, and $z) H S T /$ ACS images from GOODS HST treasury program (Giavalisco et al. 2004), and near-infrared ( $K$-band) ground-based images of the GOODS fields to select $1239 B z K$ galaxies (Daddi et al. 2007). Here, we present the results from a parametric analysis of the morphologies, using the sizes and profile shapes to quantify the morphological mix among the passive, and star-forming $B z K \mathrm{~s}$.

\section{Morphological Mix of Galaxies at $1.4 \lesssim z \lesssim 2.5$}

Our analysis of the galaxy morphology is based on fitting a simple Sérsic function (Sérsic 1968) to the two-dimensional surface brightness profiles, from which we derive the sizes, axial ratios, and the Sérsic index, $n$, which characterizes the shape of the brightness profile. We performed the analysis in the $z$-band which corresponds to the rest-frame $2900 \AA$ wavelength. We use the index, $n$, to broadly classify the galaxies as bulge-like $(n \geqslant 2.5)$, or disks and irregulars $(n<2.5)$, following the convention in previous papers (eg; Shen et al. 2003; Ravindranath et al. 2004). In Figure 2, we present the distribution of $n$ among the $B z K$-selected galaxies. Among the $B z K \mathrm{~s}$ which are tentatively classified as passive $B z K$ s because they are not detected in the $B$-band, about $40 \%$ have bulge-like profiles. However, when only the galaxies which fall well-within the region occupied by passive galaxies in the $B z K$ color-color diagram are considered, the fraction increases to almost $60 \%$. Among the star-forming $B z K \mathrm{~s}$ more than $80 \%$ have low $n$ suggesting that they are more disk-like, or irregulars including mergers.

The galaxy sizes measured by their half-light radius, $r_{e}$, also differ for the passive and star-forming samples. The distribution of sizes in Figure 2, clearly shows that for passive galaxies the peak occurs at $r_{e}=0 .{ }^{\prime \prime} 25$, with a broader distribution extending toward more compact sizes. At $z \sim 2$, this corresponds to a physical size of $2.1 \mathrm{kpc}$. On the other hand, the star-forming galaxies have larger sizes with the peak at $r_{e}=0 .{ }^{\prime \prime} 39$, which corresponds to $\sim 3.3 \mathrm{kpc}$. The smaller sizes for the passive $B z K \mathrm{~s}$ compared to those that are actively star-forming was also seen in the smaller samples analysed by Daddi et al. (2004). 

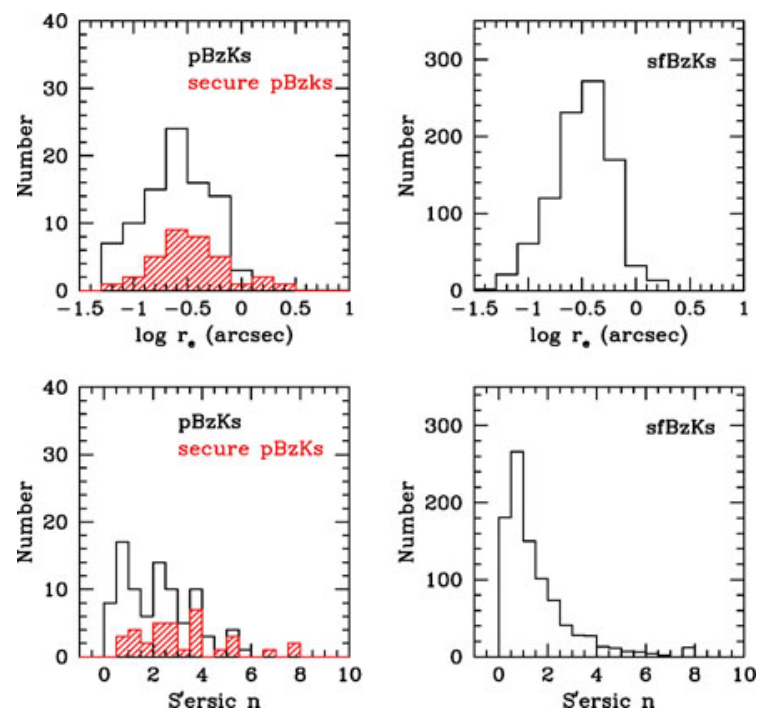

Figure 2. The distribution of the sizes, $r_{e}$, and Sérsic index, $n$, for galaxies at $1.4 \lesssim z \lesssim 2.5$ selected by the $B z K$ criteria. The distributions are shown separately for passive (left) and star-forming (right) galaxies. The red histogram is for galaxies that are unambiguously classified as passive $B z K \mathrm{~s}$, while the black histogram includes all $B z K \mathrm{~s}$ without $B$-band detection and should be considered as tentative passive $B z K \mathrm{~s}$.

\section{Population of Compact, Passively-Evolving, Massive Galaxies}

An intriguing population of galaxies are the compact, massive galaxies seen at $z \sim 2$, which have been discussed previously by Daddi et al. (2005), Trujillo et al. (2006), and Zirm et al. (2007). These are galaxies with stellar mass $>10^{10} M_{\odot}$, and sizes less than $\sim 3 \mathrm{kpc}$. At low redshifts, the galaxies of this mass in the SDSS have $r_{e}>4 \mathrm{kpc}$. In Figure 3, we show the stellar mass versus half-light radius for the $B z K \mathrm{~s}$, along with the mass-size relation for the SDSS galaxies. Clearly, most of the passively evolving galaxies from our sample are more compact than the SDSS early-type galaxies $(n>2.5)$, for the same stellar mass. The small sizes measured for the $z \sim 2$ spheroids in the $J$ and $K$ images (Trujillo et al. 2006; Zirm et al. 2007) shows that this is not merely an effect of strong morphological $k$-correction. Although, the star-forming galaxies have mean sizes that are smaller than present-day disk galaxies, there is considerable overlap at the larger sizes. Out of the 92 passive $B z K$ s in our sample, 37 galaxies have $M_{*}>10^{11} M_{\odot}$ of which 21 galaxies $(>50 \%)$ of them have sizes less than $r_{e}<3 \mathrm{kpc}$.

The bulges in the SDSS whose total stellar mass is $M_{*}=10^{11} M_{\odot}$, have a mean size of $4.2 \mathrm{kpc}$. For passive $B z K \mathrm{~s}$ with similar masses, it is $1.9 \mathrm{kpc}$, implying that the internal stellar mass density within $r_{e}$ is about 10 times larger than the SDSS galaxies. If these compact galaxies were to evolve into present-day ellipticals via gas-rich mergers, this would tend to increase the stellar mass density through dissipative process which lead to compact remnants, and the new star formation that adds to the stellar mass (Robertson et al. 2006). Therefore, the likely evolutionary scenario seems to involve "dry mergers" (Khochfar \& Burkert 2003; Khochfar \& Silk 2006). In this case, the size evolution is predicted to be around 1.25 times from $z \sim 2$ to present epoch for galaxies between $10^{10}$ and $10^{11} M_{\odot}$ and $\approx 4$ times for $5 \times 10^{11} M_{\odot}$ (Khochfar \& Silk 2006). We find that for the passive $B z K \mathrm{~s}$ with $10<\log \left(\mathrm{M} / \mathrm{M}_{\odot}\right)<11$, the mean sizes are $2.35 \pm 1.75 \mathrm{kpc}$ and comparison with the SDSS galaxies of same mass suggests at least a factor of 1.25 growth since $z \sim 2$. On the other hand, for the more massive galaxies with $\log \left(\mathrm{M} / \mathrm{M}_{\odot}\right)>11$ we 

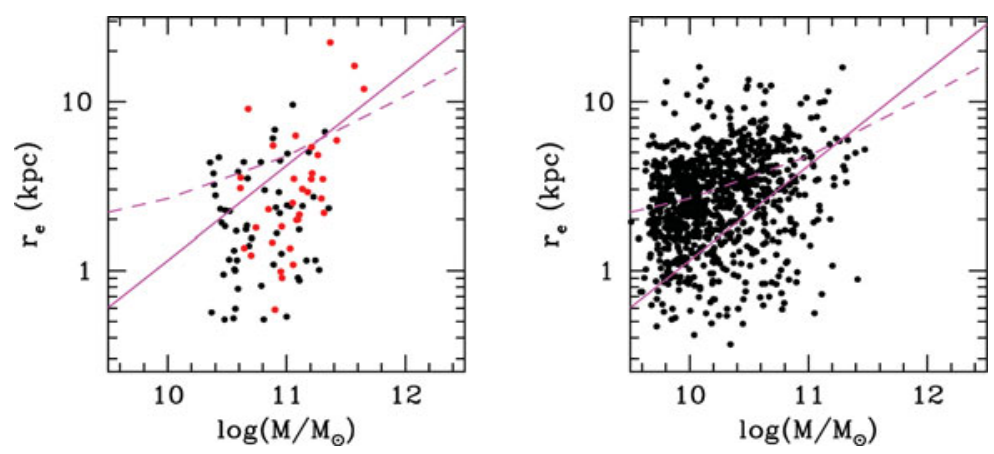

Figure 3. The half-light radius versus the Stellar mass for the passive (left panel) and starforming $B z K$ s galaxies (right panel). The magenta lines that are overplotted are the stellar mass versus size relation for the early-type galaxies (solid line) and late-type galaxies (dashed line) at $z \sim 0.1$ from the SDSS (Shen et al. 2003).

find a mean size of $3.05 \pm 1.98 \mathrm{kpc}$, which implies a factor of 2 size evolution at $z<2$. Thus we do find that qualitatively, the size evolution is more significant for the more massive galaxies.

\section{Conclusions}

Our results show that the $B z K$-selection is a more powerful technique in terms of identifying both the passive, as well as star-forming galaxies at high redshifts in order to reveal the entire range of morphological diversities. The passive $B z K \mathrm{~s}$ are mostly spheroid-like, while the star-forming galaxies are mostly disks or mergers at $z \sim 2$. Also, using a larger sample we confirm earlier reports that the high- $z$ galaxies are more compact for a given stellar mass, and it appears that they are likely to evolve to presentday ellipticals via dry mergers.

\section{References}

Lilly, S. et al. 1998, ApJ 500, 75

Stanford, S. A., et al. 2004, AJ 127, 131

Ravindranath, S., et al. 2004, ApJ 604, L9

Barden, M., et al. 2005, ApJ 635, 959

Giavalisco, M., Steidel, C. C., \& Macchetto, D. F. 1996, ApJ 470, 189

Lotz, J. M., Madau, P., Giavalisco, M., Primack, J., \& Ferguson, H. C. 2006, ApJ 636, 592

Ravindranath, S., et al. 2006, ApJ 652, 963

Giavalisco, M., et al. 2004, ApJ 600, L93

Franx, M., et al. 2003, ApJ 587, 79

Daddi, E., et al. 2004, ApJ 617,746

Daddi, E., et al. 2007, arXiv:0705.2831

Sérsic, J. L. 1968, Atlas de Galaxias Australes, (Córdoba: Obs. Astron., Univ. Nac. Córdoba)

Shen, S., et al. 2003, MNRAS 343, 978

Daddi, E., et al. 2005, ApJ 626, 680

Trujillo, I., et al. 2006, MNRAS 373, 36

Zirm, A., et al. 2007, ApJ 656, 66

Robertson, B., et al. 2006, ApJ 645, 986

Khochfar, S. \& Burkert, A. 2003, ApJ 597, 117

Khochfar, S. \& Silk, J. 2006, ApJ 648, 21 